University of Windsor

Scholarship at UWindsor

\title{
The mediating role of cultural coping behaviours on the relationships between academic stress and positive psychosocial well-being outcomes
}

\author{
B.C.H Kuo \\ University of Windsor \\ Kendall M. Soucie \\ Siqi Huang \\ Refa Laith
}

Follow this and additional works at: https://scholar.uwindsor.ca/psychologypub

Part of the Multicultural Psychology Commons

\section{Recommended Citation \\ Kuo, B.C.H; Soucie, Kendall M.; Huang, Siqi; and Laith, Refa. (2017). The mediating role of cultural coping behaviours on the relationships between academic stress and positive psychosocial well-being outcomes. International Journal of Psychology, 1-10. \\ https://scholar.uwindsor.ca/psychologypub/52 \\ This Article is brought to you for free and open access by the Department of Psychology at Scholarship at UWindsor. It has been accepted for inclusion in Psychology Publications by an authorized administrator of Scholarship at UWindsor. For more information, please contact scholarship@uwindsor.ca.}




\title{
The mediating role of cultural coping behaviours on the relationships between academic stress and positive psychosocial well-being outcomes
}

\author{
Ben C. H. Kuo, Kendall M. Soucie, Siqi Huang, and Refa Laith \\ Department of Psychology, University of Windsor, Windsor, Ontario, Canada
}

\begin{abstract}
$W$ hile culture's effect on the coping process has long been acknowledged in the stress-coping literature conceptually, empirical evidence and attempts to discern the specific relationship between culture and coping remain very scarce. Against this backdrop, the present study applied the Cultural Transactional Theory (Chun, Moos, \& Cronkite, 2006) to examine the mediating role of cultural coping behaviours (Collective, Engagement and Avoidance Coping) on the relationship between academic stress (AS) and two positive psychosocial well-being outcome measures: Collective Self-esteem (CSE) and Subjective Well-being (SWB). Responses from a sample of undergraduate students in Canada $(N=328)$ were analysed to test a theory-driven, hypothesised model of coping using structural equation modelling (SEM). As hypothesised, the SEM results showed that: (a) the proposed cultural coping model fit the data well; (b) Engagement Coping and Collective Coping partially mediated the association between AS and the outcomes and (c) the path relationships among the constructs were in the hypothesised directions. A set of preliminary exploratory analyses indicated that Collective Coping was most strongly endorsed by the African/Black and the Middle Eastern cultural groups as compared to other ethnic groups. Implications of the study's findings for future research and practice concerning culture, stress, and coping are discussed.
\end{abstract}

Keywords: Cultural coping; Collective coping; Academic stress; Collective Self-Esteem; Subjective Well-Being.

It has been widely acknowledged that culture plays a critical role in affecting the nature and process of stress and coping for individuals and groups (Lazarus \& Folkman, 1984). However, systematic scholarly efforts to articulate the relationships between cultural influences and the stress-coping process have been observed only very recently (Wong \& Wong, 2006). This emerging body of cultural-coping literature has begun to unpack the cultural-psychological dimensions that underpin coping processes and preferences among different racial and ethnic groups (Kuo, 2011; Utsey, Adams, \& Bolden, 2000). In this article, the term "cultural coping" will refer to a wide range of cognitive and affective stress responses that reflect the cultural characteristics and influences of racial or ethnic groups; examples from the literature include Africentric coping among African Americans (Utsey et al., 2000) and forbearance coping for Asians (Yeh, Arora, \& Wu, 2006). This corpus of research has pointed to individualism-collectivism, independent and interdependent self-construals and acculturation as examples of broad cultural constructs/dimensions that drive and shape the preference, pattern and process of coping among racially, ethnically and nationally diverse groups and individuals (e.g. Chun, Moos, \& Cronkite, 2006; Kuo, 2011).

In particular, the phenomenon of "Collective Coping (CC)" or "collectivistic coping" has been discussed in the cultural coping research as the intersection between collectivism, as a broad cultural value orientation, and stress-coping responses (Kuo, Roysircar, \& Newby-Clark, 2006; Yeh et al., 2006). CC behaviours have been operationalised as stress responses that involve others in culturally meaningful and appropriate ways, consider the impact of one's coping behaviours on important others, and reference culturally appropriate norms (Kuo, 2013; Yeh et al., 2006). Conceptually, this notion of relationally, communally and collectively oriented coping behaviours is supported by the prevailing cultural theories of stress-coping (Chun et al., 2006). While CC has also been found in White European participants (Kuo, Arnold, \& Rodriguez-Rubio, 2014), its prevalence and salience are especially profound among Asian Americans and Asian Canadians (e.g. Kuo et al., 2006; Wei, Hepp-

Correspondence should be addressed to Ben C. H. Kuo, Department of Psychology, University of Windsor, 401 Sunset Ave., Chrysler Hall South, Windsor, Ontario N9B 3P4, Canada. (E-mail: benkuo@uwindsor.ca). 
ner, Ku, \& Liao, 2010), and African Americans (Utsey et al, 2000).

Despite the growing evidence for culturally derived coping behaviours (e.g. CC), to the authors' knowledge, there is currently no published research that has simultaneously examined the effects of cultural coping behaviours on multiple outcome variables using cultural coping measures. Similarly, we are not aware of any published studies that have focused on the role of cultural coping in responding to academic stress (AS) specifically. Furthermore, an emerging positive theory of stress-coping (Folkman \& Moskowitz, 2004) points to the potential salutary side of the stress-coping process in terms of improving psychosocial well-being. Yet this process has also not been sufficiently examined within the current stress-coping literature.

Therefore, the purpose of the present study is to examine the role of cultural coping behaviours in response to AS in a sample of undergraduate students $(N=328)$ in Canada. Specifically, the present study aims to extend and improve upon the current cultural-coping literature by: (a) testing a hypothesised, theory-guided mediational model of cultural coping with AS based on the Cultural Transactional Theory (CTT; Chun et al., 2006) using structural equation modelling (SEM); and (b) assessing the differential effects of three coping mediators, Collective, Engagement and Avoidance Coping (as measured by the Cross-Cultural Coping Scale [CCCS] by Kuo et al., 2006) on two positive, non-symptomatic well-being indicators, Collective Self-Esteem (CSE) and Subjective Well-being (SWB). Additionally, in a series of exploratory analyses, we evaluate the differential effect of ethnic group membership on coping strategies within the context of the larger stress-coping model proposed above.

\section{Theoretical framework of the current study}

The CTT posits that collectivism and individualism represent deep-seated cultural forces, and exert broad influences over the entire process of stress-coping for both individuals and groups (Chun et al., 2006). The fundamental tenets of the CTT are closely aligned with the conceptualisation of CC behaviours described above. Specifically, the CTT stipulates that the stress-coping process is characterised by "transactions" among five mutually influencing "panels" or systems: (a) Panel I - the environmental system; (b) Panel II - the personal system; (c) Panel III-transitory conditions (e.g. life events including life stressors); (d) Panel IV - cognitive appraisal and coping skills or strategies and (e) Panel $\mathrm{V}$-health and well-being (e.g. psychosocial functioning and outcome; see Chun et al., 2006 for a detailed discussion of the CTT). In other words, the CTT posits that under stress a person's environmental and individual/personal factors (Panels I and II) will bear influences on his/her experience with a particular life event/stressor (Panel III), such as AS in the present study. In turn, this individual will appraise the impact of the stressor (e.g. AS) and respond to it with certain coping behaviours, such as Collective, Engagement and Avoidance Coping, as proposed and assessed in the current study. Finally, the effects of these preceding processes will influence the individual's health and well-being, which were measured in terms of CSE and SWB in this research (see Figure 1).

\section{Academic stress and coping}

AS is broadly defined as a disturbance induced by a student's appraisal of school-related stressors-a distress that is often manifested through psychological and somatic symptoms (Leung, Yeung, \& Wong, 2010). School burnout, as a specific form of AS, occurs when school-related demand on a student exceeds his/her resources to meet such a demand for a prolonged period of time (Salmela-Aro, Kiuru, Leskinen, \& Nurmi, 2009). School burnout is operationalised as "a syndrome of emotional exhaustion, cynicism or depersonalisation and reduced professional efficacy" (Salmela-Aro et al., 2009, p. 48). Recent studies have linked general AS as well as school burnout to coping. For instance, a recent cross-cultural study found that problem-solving coping and social support significantly mediated the link between AS and suicidal ideation in both Indian and Malaysian college students (Khan, Hamdan, Ahmad, Mustaffa, \& Mahalle, 2015). When investigated AS in terms of school burnout specifically, a recent study with Chinese middle school students in China also found the mediating effects of coping on AS (Luo, Wang, Zhang, Chen, \& Quan, 2016); task-oriented coping was negatively related to school burnout, while emotion-oriented coping was positively related to school burnout.

\section{Cultural coping behaviours: Conceptualisation and functionality}

Recent studies have identified and examined cultural coping approaches in multicultural populations across various stressful situations. For example, cultural coping behaviours have been found when responding to racial discrimination among Asian Americans (Wei, et al., 2010) and to the aftermath of the 9/11 terrorist attacks among Asian, African and Latin Americans (Constantine, Alleyne, Caldwell, McRae, \& Suzuki, 2005). Across these studies cultural coping behaviours have been characterised, operationalised, and measured with a broad repertoire of coping responses that included cultural value-based coping strategies (e.g. forbearance, familism), in-group norm referencing coping approaches (e.g. value/norm conforming responses), interpersonally-based coping methods (e.g. family 


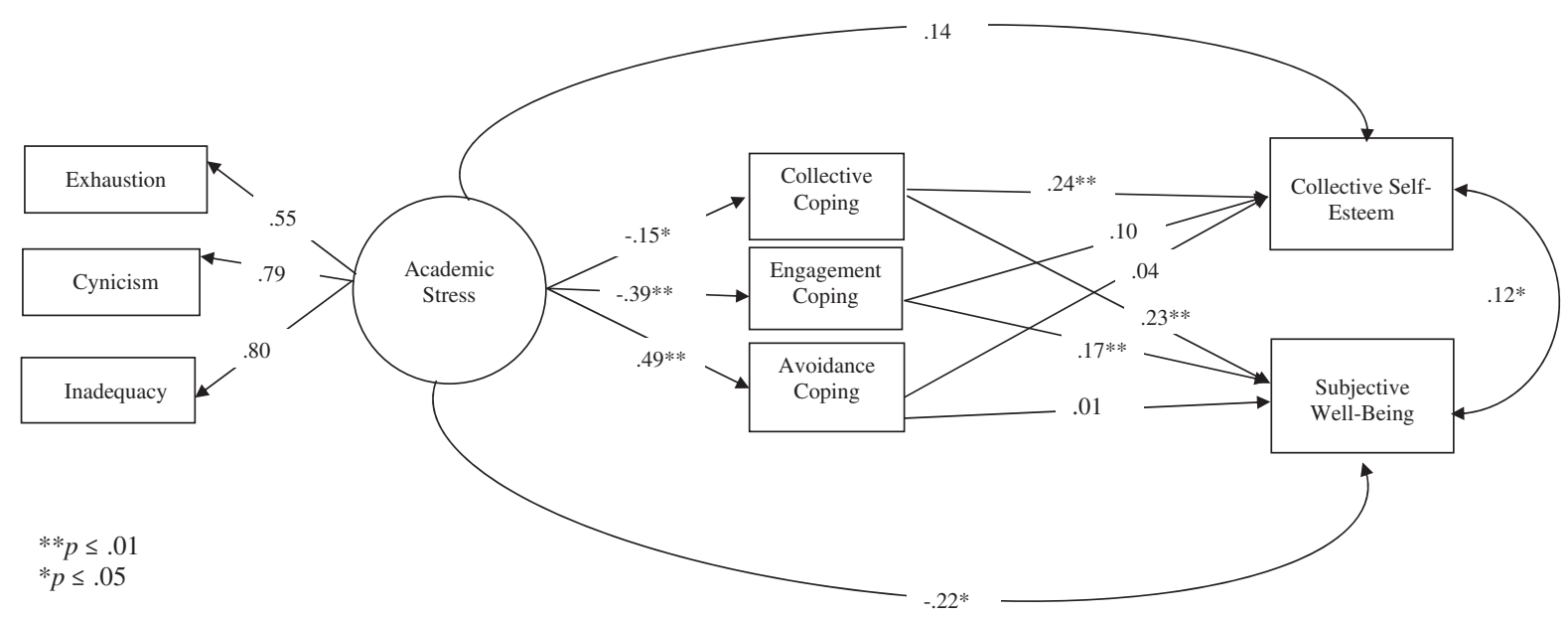

Figure 1. The hypothesised mediation model of cultural coping with the entire sample $(N=328)$. Note: All coefficients reported in the model are standardised. The path from academic stress (AS) to exhaustion was fixed to set the scale for the latent variable of AS. Path coefficients for ethnicity are not shown but were controlled for in the model. Standardised path coefficients for ethnicity on endogenous variables are as follows: Collective Coping $(\beta=-0.076)$, Engagement Coping $(\beta=0.033)$, Avoidance Coping $(\beta=-0.017)$, CSE $\left(\beta=0.106^{*}\right)$, and SWB $(\beta=-0.197 * *)$. It is important to note that these values should not be substantively interpreted as ethnicity was included as a multinomial control variable. ${ }^{*} p \leq .05$. $* * p \leq .01$.

support), culturally conditioned cognitive and emotional stress responses (e.g. acceptance, detachment, reframing, etc.) and indigenous, religious and spiritual coping strategies (Kuo, 2013; Utsey, Adams, \& Bolden, 2000). These culturally shaped coping behaviours are distinctive from intrapersonally-based, problem-focused coping (e.g. Engagement Coping [EC]) and emotion-focused coping (e.g. Avoidance Coping [AC]) that are often conceptualised and assessed in the extant-coping literature (see Kuo et al., 2006 for a review).

Research has begun to shed light on the roles cultural coping strategies play in the stress-coping process. For instance, to assess the effects of cultural coping responses, as measured by the CCCS (Kuo et al., 2006), Wester et al. (2006) conducted a model-testing study to explore the mediational effects of Collective, Engagement and Avoidance Coping on the relationship between male gender conflict and psychological distress among Chinese Canadian adolescents. Using SEM, this mediational coping model was supported by the data. However, while EC negatively predicted distress, and AC positively predicted distress, $\mathrm{CC}$ did not predict distress in the sample. A later study by Kuo et al. (2014) found that the same three CCCS cultural coping strategies mediated the link between intrinsic spirituality and psychological distress in a sample of ethnically diverse undergraduate participants. Interestingly, even though the path between CC and psychological distress was negative and non-significant, CC was positively related to the participants' level of intrinsic spirituality. In another recent study using the CCCS, Lowinger et al. (2016) found that $\mathrm{CC}$ was a negative predictor and $\mathrm{AC}$ was a positive predictor of academic procrastination in a sample of Asian international students in the USA.
Despite the evidence above, there is currently very little information about the psychosocial effects of cultural coping behaviours on broader outcomes of health, such as positive, non-symptomatic and/or socially/group-oriented indicators of well-being (Kuo et al., 2014). Incidentally, a few past studies conducted in the USA have suggested a potential link between coping and CSE and between coping and SWB among racially and ethnically diverse youth (Constantine, Donnelly, \& Myers, 2002; Vera et al, 2012). These empirical studies and the positive theory of coping (Folkman \& Moskowitz, $2000,2004)$ influenced the current study, which incorporated measures of CSE and SWB and tested the effect of coping behaviours on these two outcomes concurrently in the proposed model.

\section{THE PRESENT STUDY}

Grounded in the CTT (Chun et al., 2006), the present study sought to examine the mediating role of cultural coping behaviours on the relationship between AS and two outcome measures: SWB and CSE (see Figure 1). The competing effects of Collective, Engagement and Avoidance Coping were simultaneously examined in this study, because the former represents understudied coping behaviours that are intimately shaped by collectivistic cultural values, whereas the latter two represent widely researched coping strategies that correspond to problem-focused coping and emotion-focused coping in the extant stress-coping literature (Kuo, et al., 2006). Accordingly, the following research question was posed: Does Collective, Engagement, and Avoidance Coping mediate the relationship between AS, as the stress 
precursor, and CSE and SWB, as the sociocultural and psychological outcomes?

On the basis of previous research involving the CCCS (e.g. Lowinger et al., 2016; Wester, Kuo \& Vogel, 2006), we hypothesised that undergraduate participants' AS would be mitigated by their use of CC and EC (see Figure 1). As such, negative paths/relationships between AS and the two types of coping behaviours were expected. $\mathrm{AC}$ was predicted to exacerbate AS and thus it would form a positive path with AS (Kuo et al., 2014). In turn, both $\mathrm{CC}$ and $\mathrm{EC}$ were postulated to form positive relationships with both CSE and SWB, but AC was predicted to form negative relationships with the same outcome variables.

\section{METHODS}

\section{Participants}

All 328 participants $(81.4 \%$ female, $18.6 \%$ male; mean age $=21.06, S D=4.48)$ in the current study were recruited from the Psychology Participant Pool of a mid-size university in Ontario, Canada. All participants read and signed an informed consent form prior to completing a paper-and-pencil questionnaire containing demographic questions and several measures. The sample was comprised of $62.5 \%$ White Europeans, $7.3 \%$ Africans/Blacks, 6.7\% Middle Eastern, 8.2\% South Asians, 5.8\% East Asians, 1.8\% Native/First Nation, $0.3 \%$ Hispanics; $7.3 \%$ identified themselves in the "other" category. A total of $50.3 \%$ of the participants reported as being third generation immigrants or beyond, $27.1 \%$ as second generation, $13.4 \%$ as 1.5 generation (born outside of Canada but immigrated before age 12), $6.4 \%$ as first generation (born outside of Canada and immigrated at or after age 12) and $2.4 \%$ as foreign-born international students.

\section{Measures}

\section{The School Burnout Inventory}

The School Burnout Inventory (SBI) is a nine-item questionnaire that assesses three components of AS in terms of respondents' feeling and perception of exhaustion with schoolwork, cynicism toward the meaning of school, and sense of inadequacy at school (Salmela-Aro et al., 2009). Participants were asked to rate each item from 1 (completely disagree) to 6 (strongly agree), with higher scores denoting greater AS. The scale's Cronbach's alpha for the current study was .829 .

\section{The Cross-Cultural Coping Scale}

The CCCS is a 27-item, scenario-based coping scale that assesses three types of coping approaches, CC, EC and AC; Kuo et al., 2006). The scale first directed respondents to read a hypothetical stressful scenario-in the present study, a vignette depicting significant AS. The scenario describes a university student facing a significant amount of stress due to poor grades, overwhelming schoolwork demands, and significant concern about the student's academic future. Participants then rated each of the coping items from 1 (very inaccurate) to 6 (very accurate) by responding to how they would cope with the stressful situation described in the scenario. Higher scores on each of the coping subscales represent a greater utilisation of the corresponding coping strategies. In the present study, the Cronbach's alphas were .800 for the CC subscale, .689 for the EC subscale and .651 for the AC subscale.

\section{The Collective Self-Esteem Scale}

The 16-item Collective Self-Esteem Scale (CSES; Luhtanen \& Crocker, 1992) consists of four specific domains of social self-esteem: membership, private, public, and identity. Respondents are asked to rate statements describing positive and negative feelings and beliefs about their group memberships (e.g. related to race, religion and ethnicity) from 1 (strongly disagree) to 7 (strongly agree), with higher scores presenting stronger identification with one's social group. In the present research, the CSES was employed to assess participants' cultural identity in terms of race or ethnicity as did in previous research (e.g. Constantine et al., 2002). For this study, an overall CSES score was used in the subsequent analyses, with higher scores representing stronger identification with one's racial and ethnic group. The overall CSES Cronbach's alpha in the present study was .789 .

\section{Satisfaction with Life Scale}

The 5-item Satisfaction with Life Scale (SWLS) is a brief instrument developed by Diener, Emmons, Larsen, \& Griffin (1985) to assess respondents' SWB in terms of their global satisfaction with life. Respondents indicate their agreement to the five statements based on their perception of their health and wellness on a 7-point Likert, from 1 (strongly disagree) to 7 (strongly agree). Higher scores on the scale signify greater perceived overall wellbeing. In this study, the Cronbach's alpha for SWLS was .852.

\section{RESULTS}

\section{Preliminary analyses}

Table 1 provides the descriptive statistics for all variables tested in the proposed stress-coping mediation model. Table 2 provides the intercorrelations among all study variables. Bivariate correlational analyses indicated that 
TABLE 1

Descriptive statistics for academic stress, cultural coping behaviours, Collective Self-Esteem (CSE) and Subjective Well-being (SWB)

\begin{tabular}{lccc}
\hline Variable & $M$ & $S D$ & Range \\
\hline EXH & 3.70 & 1.06 & $1-6$ \\
CYN & 3.16 & 1.30 & $1-6$ \\
INA & 3.38 & 1.25 & $1-6$ \\
CC & 3.36 & .96 & $1-6$ \\
AC & 3.06 & .66 & $1-6$ \\
EC & 4.63 & .66 & $1-6$ \\
CSE & 4.93 & .62 & $1-7$ \\
SWB & 4.82 & 1.24 & $1-7$ \\
\hline
\end{tabular}

Note: $\mathrm{EXH}=\mathrm{SBI}$ exhaustion; $\mathrm{CYN}=\mathrm{SBI}$ cynicism; INA = SBI inadequacy; $\mathrm{CC}=$ Collective Coping; $\mathrm{AC}=$ Avoidance $\quad$ Coping; $\mathrm{EC}=$ Engagement $\quad$ Coping; $\quad \mathrm{CSE}=$ Collective $\quad$ Self-Esteem; SWB $=$ Subjective Well-being

TABLE 2

Intercorrelations of academic stress, cultural coping behaviours, CSE and SWB for the sample

\begin{tabular}{|c|c|c|c|c|c|c|c|c|}
\hline & EXH & $C Y N$ & INA & $C C$ & $A C$ & $E C$ & CSE & $S W B$ \\
\hline EXH & 1.00 & & & & & & & \\
\hline CYN & $.44 * *$ & 1.00 & & & & & & \\
\hline INA & $.45^{* *}$ & $.63^{* *}$ & 1.00 & & & & & \\
\hline $\mathrm{CC}$ & -.04 & $-.13^{* *}$ & $-.09 \dagger$ & 1.00 & & & & \\
\hline $\mathrm{AC}$ & $.24 * *$ & $.40^{* *}$ & $.38^{* *}$ & $-.12 * *$ & 1.00 & & & \\
\hline $\mathrm{EC}$ & $-.19 * *$ & $-.29 * *$ & $-.33 * *$ & $.22 * *$ & $-.17 * *$ & 1.00 & & \\
\hline CSE & $-.17 * *$ & $-.24 * *$ & $-.22 * *$ & $.43^{* *}$ & -.09 & $.21 * *$ & 1.00 & \\
\hline SWB & $-.20 * *$ & $-.30^{* *}$ & $-.23 * *$ & $.32 * *$ & $-.16^{* *}$ & $.30^{* *}$ & $.37 * *$ & 1.00 \\
\hline $\begin{array}{l}\text { Note: } \\
\text { inadec } \\
\text { ing; } \\
\text { SWB } \\
\text { **p } \leq\end{array}$ & $\begin{array}{l}\mathrm{EXH}= \\
\text { quacy; } \\
\mathrm{EC}=\mathrm{Er} \\
=\text { Subjec } \\
.01 . * p \leq\end{array}$ & $\begin{array}{l}=\mathrm{SBI} \\
\mathrm{CC}=\mathrm{C} \\
\text { ingageme } \\
\text { ctive We } \\
\leq .05 . \dagger p\end{array}$ & $\begin{array}{l}\text { xhaustion } \\
\text { Collective } \\
\text { ent Cop } \\
\text { ll-being. } \\
<.08 \text {. }\end{array}$ & $\begin{array}{l}\text { n; CYN } \\
\text { e Copi } \\
\text { ping; }\end{array}$ & $\begin{array}{l}\mathrm{N}=\mathrm{SBI} \\
\text { ing; } \\
\mathrm{CSE}=\mathrm{Co}\end{array}$ & $\begin{array}{l}\text { cynicisn } \\
\mathrm{C}=\mathrm{Avo} \\
\text { ollective }\end{array}$ & $\begin{array}{l}\mathrm{m} \text {; IN } \\
\text { oidance } \\
\text { Self-I }\end{array}$ & $\begin{array}{l}=\mathrm{SBI} \\
\text { Cop- } \\
\text { steem; }\end{array}$ \\
\hline
\end{tabular}

relationships were in the hypothesised directions. AC was positively and significantly correlated with the three subscales of the SBI, while EC was significantly negatively related to all three subscales.

While CC showed negative relationships with AS, only the relationship between $\mathrm{CC}$ and the Cynicism subscale was significant $(p \leq .05)$. Both CC and EC were positively associated with SWB and CSE, while AC was negatively associated with both outcome variables. SWB and CSE were also positively and significantly associated with each other. These correlations indicate underlying structural relationships among these key variables in the data, which then provide sufficient common variance to test the hypothesised conceptual model with an SEM analysis.

In the analyses reported below, a full information maximum likelihood (FIML) estimation procedure was used to estimate missing values using M-plus v.5.0 (Muthén $\&$ Muthén, 2008). FIML allows for the utilisation of complete data based on all available information in the data (Graham, 2009). Goodness-of-fit was evaluated using multiple methods. These included an exact-fit chi-square Test (Hu \& Bentler, 1998), two incremental fit indices; the comparative fit index (CFI) and the non-normed fit index (NNFI) both of which range from 0.00 to 1.00 with 1.00 indicating a perfect model fit ( $\mathrm{Hu} \&$ Bentler, 1998), and the root mean square error of approximation (RMSEA) with values less than .08 indicating adequate model fit (Hu \& Bentler, 1998). All path coefficients are reported as standardised estimates.

\section{Testing the overall hypothesised model of cultural coping}

First, AS was defined as a latent construct comprised of the three subscales of the SBI and was specified as an exogenous predictor of the three coping strategies. The three coping behaviours were then specified to predict CSE and SWB as endogenous variables (see Figure 1). Direct paths from AS to CSE and SWB were also specified in the model and CSE and SWB were specified to be correlated, based on past research (e.g. Vera et al., 2012). The SEM results show that, controlling for ethnicity, this overall stress-coping mediation model yielded a good fit to the data, $\chi^{2}(15)=24.049, p=.06$; $\mathrm{CFI}=.98$; $\mathrm{NNFI}=.96 ; \quad \mathrm{RMSEA}=.04 ; \quad 95 \% \mathrm{CI}=[.000-.073]$. Ethnicity was controlled for by including a single, seven-category multinomial ethnicity variable in the model (comprised of each of the seven ethnicities reported above with the exception of "other") with paths from ethnicity to all five endogenous variables. As such, the model paths reflect variance accounted for above and beyond ethnicity. We did not include paths in the figure of the overall model for the purpose of clarity and because the multinomial nature of the variable precludes interpretation of the path coefficients beyond variance explained. However, we include the path coefficients as a footnote in Figure 1.

As shown in Figure 1, the direct path from AS to CSE was not significant. However, the direct path from AS to SWB was significant, such that higher scores on AS were associated with lower scores on SWB. The paths from AS to all three coping variables were also statistically significant and in the hypothesised directions. As expected, higher AS scores were associated with lower scores on both CC and EC; however, higher AS scores were associated with higher scores on AC. Further, higher levels of CC were positively associated with CSE and SWB, but EC was only positively associated with SWB. AC was not associated with CSE or SWB. CSE and SWB were significantly positively associated. The model also accounted for a significant percentage of variance in the endogenous outcome variables. The significant $R^{2}$ values were .08 and .24 for CSE and SWB, respectively. 
TABLE 3

Significance tests for direct and indirect effects from academic stress (AS) to CSE and SWB

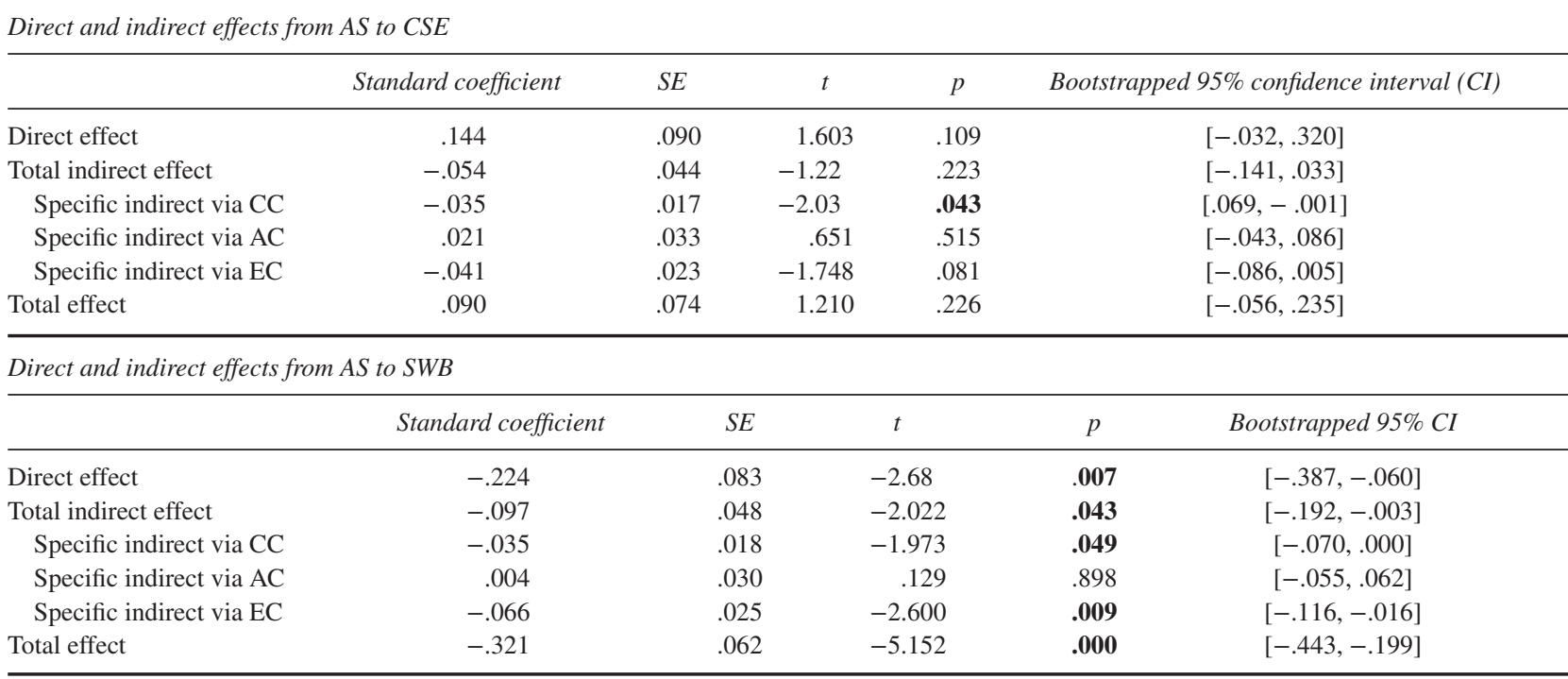

Note: $\mathrm{CC}=$ Collective Coping; $\mathrm{AC}=$ Avoidance Coping; $\mathrm{EC}=$ Engagement Coping; $\mathrm{CSE}=$ Collective Self-Esteem; $\mathrm{SWB}=$ Subjective Well-being. The bolded values represent $p$-values that are <.05.

\section{Testing for significant indirect effects}

Indirect effects were calculated using Mplus v.5.0 and the standard errors for the indirect effects were estimated with a bootstrapping procedure using 1000 bootstrapped samples (Little, Card, Bovaird, Preacher, \& Crandall, 2007). The ratio of the indirect effect coefficient to its corresponding standard error yields a $t$-value which was used to determine significance (a ratio of $b / b_{\text {s.e. }}>1.96$ is significant at the .05 level in a large sample). Results from the bootstrapping method indicated that the total indirect effect from AS to CSE was not significant; however, there was a significant specific indirect path from AS to CSE via CC. None of the other specific indirect paths were significant for CSE. The total indirect effect from AS to SWB was significant. There was a significant specific indirect effect from AS to SWB via EC and a marginally significant effect through CC. Therefore, a significant amount of the variance between AS and the two outcome variables is accounted for by the mediating coping variables. The complete set of standardised direct and indirect effect coefficients and corresponding 95\% confidence intervals (CI) are provided in Table 3.

\section{Exploratory analyses: Testing the hypothesised model of cultural coping with ethnic group predictors}

Following the finding of an overall model fit, we conducted a series of follow-up SEM analyses which included ethnicity as a set of dummy coded variables for each of the five largest ethnic subgroup in the sample:
White European, African/Black, Middle Eastern, East Asian, and South Asian. These variables were included as manifest predictors of both AS and CC, because preliminary analyses revealed that there were no significant differences among these five ethnic groups on either EC or AC. The models were estimated separately for each ethnic group to make interpretation of the findings more clear (see Appendix A). Interestingly, while there were no ethnic group differences in the use of $\mathrm{EC}$ and $\mathrm{AC}$, the use of CC was most salient among African/Black and Middle Eastern participants in dealing with AS. These between-group findings, though notable and consistent with our theoretical predictions, should be treated as highly tentative and interpreted with caution. These analyses are considered to be exploratory because of the unequal sample size across these groupings, ranging from 205 for White Europeans to 19 for East Asians (see Table A1 in Appendix A).

\section{DISCUSSION}

The findings of this present study extend the current literature on cultural coping research by offering a number of important and novel insights. First, the proposed mediational model of coping in the study was supported by the SEM analysis, as hypothesised. The model indicates that individuals reporting the highest levels of AS also reported the lowest levels of utilisation of CC and EC. Conversely individuals reporting high levels of AS reported higher levels of using AC coping methods. This is consistent with the notion that utilising CC and EC strategies may mitigate the impact of undergraduate 
students' AS on CSE and SWB, while using AC either did not reduce or possibly could aggravate AS. These findings align with results from previous studies. For example, Wester et al. (2006) found that the use of CC was related to less restrictive emotionality, a form of stress stemming from male gender conflict, among Chinese Canadian male adolescents. Similarly, Lowinger et al. (2016) found that the use of CC predicted lower levels of academic procrastination, a behavioural manifestation of underlying AS, among Asian international students in the US. The current findings are also consistent with the hypothesised beneficial nature of EC (as a form of adaptive problem-solving coping) and the maladaptive quality of $\mathrm{AC}$ (as a form of unproductive emotion-focused coping). These results are in line with the findings of previous studies focusing on coping and school burnout (e.g. Luo et al., 2016), and cultural coping research using the CCCS (e.g. Kuo et al., 2014; Lowinger et al., 2016).

Secondly, the SEM results point to differential patterns of mediational effects on psychosocial well-being indicators across the three coping approaches. The negative indirect effects between AS and CSE and SWB via $\mathrm{CC}$ and $\mathrm{EC}$ are commensurate with the hypothesis that those students reporting higher levels of AS also report lower levels of effective coping strategies (i.e. CC and EC) which in turn is associated with lower reported levels of CSE and SWB. In contrast, students reporting higher levels of AS reported higher levels of ineffective coping strategies (AC) which in turn was predictive of lower levels of CSE and SWB.

With respect to CSE and CC, the current findings are in line with an earlier study, which showed that African American adolescents who reported higher level of CSE with their cultural group were more likely to use Africultural collective and spiritual coping strategies (Constantine et al., 2002). It makes theoretical sense to infer that $\mathrm{CC}$ behaviours - stress responses strongly driven by values, beliefs and attitudes of one's cultural in-group - would be closely related and contribute to the individual's sense of social esteem, as well as membership and identity with respect to such a reference group (Constantine et al., 2002; Kuo, 2013). This proposition was empirically supported in the present study. It is quite possible that by engaging in $\mathrm{CC}$ behaviours in response to a stressor, an individual gains a stronger sense of solidarity with, commitment to, and perceived acceptance by his/her own in-groups, independent of whether these coping behaviours actually bring about emotional relief from the distress.

On this latter point, the non-symptomatic, psychosocial benefits of collectivistic coping behaviours through augmenting CSE and SWB might be broadly conceptualised from the emerging perspective of the positive theory of coping (Folkman \& Moskowitz, 2004). Unlike previous coping theories, the positive coping theory underscores the co-occurrence of both positive and negative effects/consequences associated with the stress-coping process. Folkman and Moskowitz observed that when faced with stress, positive affect can result from positive reappraisal that are "linked to the individual important values, beliefs and goals that comprise the individual's sense of meaning" (Folkman \& Moskowitz, 2004, p. 766) and "seeing how one's own efforts can benefit other people" (Folkman \& Moskowitz, 2000, p. 116). Both of these elements highlight the essence of $\mathrm{CC}$ as expounded in the earlier section (Kuo, 2013; Yeh et al., 2006).

Thirdly, the exploratory results presented in Appendix A suggest that this cultural coping model might have some cross-cultural generalisability. In turn, the results give empirical credence to the CTT, the theoretical scheme upon which the current research was based. It is also interesting to note that both African/Black and Middle Eastern participants reported more use of CC than other groups in dealing with AS, which may yield a greater buffering effect of CC for these two groups. Despite the sample size limitation, these relationships are consistent with prior research and theoretical predictions (Kuo et al., 2006). One possible explanation is that these two groups may have stronger and more "traditional" collectivistic values and religious beliefs than the other groups. Previously published works have found that cultural groups that are deeply religious might be more inclined to engage in collectivistically oriented coping in time of stress (Fischer, Ai, Aydin, Frey, \& Haslam, 2010; Kuo et al., 2006). This pattern applies to African Americans (Constantine et al., 2002, 2005) and Muslims worldwide (Fischer et al., 2010), among whom a significant number may be of Middle Eastern backgrounds.

\section{Limitations of the current study}

There are a number of limitations to be considered in this research. First, this investigation relied solely on cross-sectional data based on a survey design. The relationships among the variables in the hypothesised model were analysed with correlations and statistical inferences. The actual temporal and causal relationships among the model's variables cannot fully be ascertained without a longitudinal study devoted to assessing coping processes over time. Second, the level of AS reported by this current sample group was only at a moderate level $(M=3.41$ out of 6 on the SBI). Therefore, it is not clear how closely the mediational coping patterns identified in this study reflect the actual coping experiences of undergraduate students whose AS is significant to the point of necessitating actual coping responses. Thirdly, the underrepresentation of males (18.6\%) and the small sample size for the four non-White European ethnic groups in the study sample are far from ideal and are likely to have influenced the present results. Future research should 
seek more gender-balanced samples and engage in more strategic recruitment approaches to obtain representative samples.

\section{Implications for research and practice}

In term of future research directions, the current mediational coping model with AS would greatly benefit from further replication and extension. Future studies should consider extending this study by testing the cultural coping model with: (a) similar college/university sample populations in different localities; (b) different stressors, such as interpersonal conflicts, illnesses, trauma, and discrimination and (c) larger samples of ethnic, racial and international groups. Clearly, this line of research would help verify the stability and validity of the present cultural coping model across broad and diverse populations and contexts.

At the theoretical front, this study highlights the value of theory-guided research in discerning the complex intersections among culture, stress and coping. On this point, the study's model-testing results substantiate the mediational link among the stress precursor, coping mediators and health and well-being outcomes (Panels III to $\mathrm{V}$ of the CTT). Moreover, the CTT provides a conceptual basis to help explain the phenomenon of $\mathrm{CC}$ and its role in the hypothesised coping model. Similarly, in terms of measurement, this study underscores the importance of employing cultural measures of coping (such as the use the CCCS in this study) in stress-coping research involving multicultural populations. The use of culturally derived coping inventories serves to broaden the narrow individualistically and intrapersonally focused coping dimensions typically operationalised and assessed within Western psychology (Yeh et al., 2006). As evident in this study, the salience and significance of the $\mathrm{CC}$ in the current sample could only have been fleshed out with the use of a culturally sensitive coping measure, the CCCS. Considering the above, future coping research with culturally diverse populations should vigorously strive to incorporate validated cultural coping theories and measures into the design and implementation of the study (Kuo, 2011; Wong \& Wong, 2006).

Finally, the present study holds implications for counselling and psychotherapy interventions with college/university students. The cultural coping model confirms the contributive value of $\mathrm{CC}$ and EC versus the aversive impact of $\mathrm{AC}$ for students coping with school-related stress and burnout. Evidently, students should be advised to reduce or even eliminate the use of avoidant responses such as wishful thinking, escapism and diversion, and instead be encouraged to mobilise problem-focused responses along with collectivistic coping strategies in countering their academic stressors. Counsellors should be mindful that the need and tendency to resort to family and in-group as sources of coping (i.e. CC) with stress might be particularly heightened among certain cultural groups. Instead of discussing only intrapersonal coping strategies, counsellors should explore other potential coping strategies that might hold cultural or relational significance with diverse clients; such as asking the client "What might your parents/siblings/extended family/co-ethnic peers/cultural values/religious-spiritual beliefs say or suggest about how you should coping with your current stressors? How might that be important to you?" Lastly, the study's findings highlight the positive side of stress-coping. This serves as a reminder for counsellors and clients alike to gauge coping effectiveness more broadly to include the impact of coping on the enhancement of positive social esteem, acceptance and well-being (Folkman \& Moskowitz, 2004) - a perspective that transcends the physical or psychological distress paradigm of stress-coping.

\author{
Manuscript received May 2016 \\ Revised manuscript accepted February 2017
}

\section{REFERENCES}

Chun, C. A., Moos, R. H., \& Cronkite, R. C. (2006). Culture: A fundamental context for the stress and coping paradigm. In $\mathrm{P}$. T. P. Wong \& L. C. J. Wong (Eds.), Handbook of multicultural perspectives on stress and coping (pp. 29-53). New York, NY: Springer. doi:10.1007/0-387-26238-5_2

Constantine, M. G., Alleyne, V. L., Caldwell, L. D., McRae, M. B., \& Suzuki, L. A. (2005). Coping responses of Asian, Black, and Latio/Latina New York City residents following the September 11, 2001 Terrorist attacks against the United States. Cultural Diversity \& Ethnic Minority, 11(4), 293-308. doi:10.1037/1099-9809.11.4.293.

Constantine, M. G., Donnelly, P. C., \& Myers, L. J. (2002). Collective self-esteem and africultural coping styles in African American adolescents. Journal of Black Studies, 32(6), 698-710. doi:10.1177/00234702032006004.

Diener, E., Emmons, R. A., Larsen, R. J., \& Griffin, S. (1985). The satisfaction with life scale. Journal of Personality Assessment, 49(1), 71-75. doi:10.1207/s15327752 jpa4901_13.

Fischer, P., Ai, A. L., Aydin, N., Frey, D., \& Haslam, S. A. (2010). The relationship between religious identity and preferred coping strategies: An examination of the relative importance of interpersonal and intrapersonal coping in Muslim and Christian faiths. Review of General Psychology, 14(4), 365-381. doi:10.1037/a0021624.

Folkman, S., \& Moskowitz, J. T. (2000). Stress, positive emotion, and coping. Current Directions in Psychological Science, 9(4), 115-118. doi:10.1111/1467-8721.00073.

Folkman, S., \& Moskowitz, J. T. (2004). Coping: Pitfalls and promise. Annual Review of Psychology, 55(1), 745-774. doi:10.1146/annirev.psych.55.090902.141456.

Graham, J. W. (2009). Missing data analysis: Making it work in the real world. Annual Review of Psychology, 60, 549-576. doi:10.1146/annurev.psych.58.110405.085530. 
Hu, L. T., \& Bentler, P. M. (1998). Fit indices in covariance structure modeling: Sensitivity to underparameterized model misspecification. Psychological Methods, 3(4), 424-453. doi:10.1037/1082-989x.3.4.424.

Khan, A., Hamdan, A. R., Ahmad, R., Mustaffa, M. S., \& Mahalle, S. (2015). Problem-solving coping and social support as mediators of academic stress and suicidal ideation among Malaysian and Indian adolescents. Community Mental Health Journal, 52(2), 245-250. doi:10.1007/s10597-015-9937-6.

Kuo, B. C. H. (2011). Culture's consequences on coping: Theories, evidence, and dimensionalities. Journal of Cross-Cultural Psychology, 42(6), 1084-1100. doi:10.1177/0022022110381126.

Kuo, B. C. H. (2013). Collectivism and coping: Current theories, evidence, and measurements of collective coping. International Journal of Psychology, 48(3), 374-388. doi:10.1080/00207594.2011.640681.

Kuo, B. C. H., Arnold, R., \& Rodriguez-Rubio, B. (2014). Mediating effects of coping in the link between spirituality and psychological distress in a culturally diverse undergraduate sample. Mental Health, Religion and Culture, 17(2), 173-184. doi:10.1080/13674676.2013.780015.

Kuo, B. C. H., Roysircar, G., \& Newby-Clark, I. R. (2006). Development of the Cross-Cultural Coping Scale: Collective, avoidance, and engagement strategies. Measurement and Evaluation in Counseling and Development, 39, 161-181.

Lazarus, R. S., \& Folkman, S. (1984). Stress, appraisal, and coping. New York, NY: Springer.

Leung, G. S. M., Yeung, K. C., \& Wong, D. F. K. (2010). Academic stressors and anxiety in children: The role of parental support. Journal of Child and Family Study, 19(1), 90-100. doi:10.1007/s10826-009-9288-4.

Little, T. D., Card, N. A., Bovaird, J. A., Preacher, K., \& Crandall, C. S. (2007). Structural equation modeling of mediation and moderation with contextual factors. In T. D. Little, J. A. Bovaird, \& N. A. Card (Eds.), Modeling contextual effects in longitudinal studies. Mahwah, NJ: Erlbaum.

Lowinger, R. J., Kuo, B. C. H., Song, H.-A., Mahadevan, L., Kim, E., Liao, K. Y.-H., ... Han, S. (2016). Predictors of academic procrastination in Asian international college students. Journal of Student Affairs Research and Practice, 53(1), 90-104. doi:10.1080/19496591.2016.1110036.

Luhtanen, R., \& Crocker, J. (1992). A collective self-esteem scale: Self-evaluation of one's social identity. Personality and Social Psychology Bulletin, 18(3), 302-318. doi:10.1177/0146167292183006.

Luo, Y., Wang, Z., Zhang, H., Chen, A., \& Quan, S. (2016). The effect of perfectionism on school burnout among adolescence: The mediator of self-esteem and coping style. Personality and Individual Differences, 88, 202-208. doi:10.1016/j.paid.2015.08.056.

Muthén, B. O., \& Muthén, L. K. (2008). Mplus Users Guide. Los Angeles, CA: Muthén \& Muthén.

Salmela-Aro, K., Kiuru, N., Leskinen, E., \& Nurmi, J.-E. (2009). School Burnout Inventory (SBI): Reliability and validity. European Journal of Psychological Assessment, 25(1), 48-57. doi:10.1027/1015-5759.25.1.48.

Utsey, S. O., Adams, E. P., \& Bolden, M. (2000). Development and initial validation of the Africultural Coping Systems
Inventory. Journal of Black Psychology, 26(2), 194-215. doi:10.1177/0095798400026002005.

Vera, E. M., Vacek, K., Blackmon, S., Coyle, L., Gomez, K., Jorgenson, K., \& Steele, J. C. (2012). Subjective well-being in urban, ethnically diverse adolescents: The role of stress and coping. Youth \& Society, 44, 331-347. doi:10.1177/0044118x11401432.

Wei, M., Heppner, P. P., Ku, T.-Y., \& Liao, K. Y.-H. (2010). Racial discrimination stress, coping, and depressive symptoms among Asian Americans: A moderation analysis. Asian American Journal of Psychology, 1(2), 136-150. doi:10.1037/a0020157.

Wester, S. R., Kuo, B. C. H., \& Vogel, D. L. (2006). Multicultural coping: Chinese-Canadian adolescents, male gender role conflict, and psychological distress. Psychology of Men \& Masculinity, 7(2), 83-100. doi:10.1037/1524-9220.7.2.83.

Wong, P. T. P., \& Wong, L. C. J. (2006). Handbook of multicultural perspectives on stress and coping. New York, NY: Springer.

Yeh, C. J., Arora, A. K., \& Wu, K. A. (2006). A new theoretical model of collectivistic coping. In P. T. P. Wong \& L. C. J. Wong (Eds.), Handbook of multicultural perspectives on stress and coping (pp. 55-72). New York, NY: Springer.

\section{APPENDIX}

Table A1. Fit indices for the preliminary ethnicity models

\begin{tabular}{lcccccc}
\hline Model & $N$ & $d f$ & \multicolumn{1}{c}{$\chi^{2}$} & RMSEA & CFI & NNFI \\
\hline White European & 205 & 17 & 60.37 & .08 & .92 & .84 \\
Black & 24 & 17 & $31.37^{* *}$ & .05 & .97 & .94 \\
Middle Eastern & 22 & 17 & $27.74^{* *}$ & .04 & .98 & .90 \\
East Asian & 19 & 17 & $28.77^{* *}$ & .05 & .98 & .90 \\
South Asian & 27 & 17 & $23.38^{*}$ & .03 & .99 & .92 \\
\hline
\end{tabular}

RMSEA = root mean square error of approximation; $\mathrm{CFI}=$ comparative fit index; NNFI $=$ non-normed fit index.

$* * p \leq .01 . * p \leq .05$.

All five models tested provided an acceptable fit to the data. The standardised path coefficients indicated that White European participants reported significantly lower levels of AS $(\beta=-.16, p=.041)$ but did not significantly differ from other ethnic groups in the use of $\mathrm{CC}$ strategies. African/Black participants did not differ significantly from other ethnic groups in their levels of AS, but did report marginally significantly higher levels of CC $(\beta=.10, p=.07)$. Similarly, the Middle Eastern participants also did not differ from other ethnic groups with respect to AS, but did report significantly higher levels of CC $(\beta=.15, p=.004)$. South Asian and East Asian participants did not differ significantly from each other or other ethnic groups in their reported AS or their usage of CC. The relationships between AS and the three cultural coping behaviours, as well the mediational effects of the coping strategies on CSE and SWB, were consistent with the patterns identified when the coping model was tested with the full sample. Interestingly, while there were no ethnic group differences in the use of EC and AC, 
the use of CC was most salient among African/Black and Middle Eastern participants in dealing with AS. Again, even though the use of dummy-coded predictors allowed for the full sample to be included in the estimation of the model, the proportions of ethnic groups relative to the entire sample were small (i.e. all under 10\%). These between-group findings, though notable and consistent with our theoretical predictions, should be treated as highly tentative and interpreted with caution. 